\title{
A Prospective Observational Survey on the Long-Term Effect of LDL Apheresis on Drug-Resistant Nephrotic Syndrome
}

\author{
Eri Muso $^{\text {a }}$ Masatoshi Mune ${ }^{d}$ Tsutomu Hirano ${ }^{e}$ Motoshi Hattori $^{g}$ Kenjiro Kimurak \\ Tsuyoshi Watanabel Hitoshi Yokoyama ${ }^{m}$ Hiroshi Sato $^{\mathrm{n}}$ Shunya Uchida ${ }^{\mathrm{h}}$ \\ Takashi Wada $^{\circ}$ Tetsuo Shoji ${ }^{b}$ Tsukasa Takemura ${ }^{c}$ Yukio Yuzawa ${ }^{p}$ Satoru Ogahara ${ }^{q}$ \\ Satoshi Sugiyama $^{\text {s }}$ Yasuhiko Iino ${ }^{i}$ Soichi Sakaij Yousuke Ogura ${ }^{f}$ Susumu Yukawa ${ }^{4}$ \\ Yoshiki Nishizawa $^{\mathrm{b}}$ Noriaki Yoriokav $^{\mathrm{E}}$ Enyu Imaiw ${ }^{\mathrm{w}}$ Seiichi Matsuo ${ }^{\mathrm{t}}$ Takao Saito $^{\mathrm{r}}$
}

a Division of Nephrology and Dialysis, Kitano Hospital, Tazuke Kofukai Medical Institute, ${ }^{b}$ Department of Metabolism, Endocrinology and Molecular Medicine, Osaka City University Graduate School of Medicine, and 'Department of Pediatrics, Kinki University School of Medicine, Osaka, d Ryoshukai Takaishi Fujii Hospital, Takaishi, e Department of Diabetes, Metabolism and Endocrinology, and ${ }^{\mathrm{f}}$ Division of Nephrology, Department of Medicine, Showa University School of Medicine, ${ }^{9}$ Department of Pediatric Nephrology, Tokyo Women's Medical University, ${ }^{\text {h }}$ Department of Internal Medicine, Teikyo University School of Medicine, 'Department of Nephrology, Nippon Medical School, and ${ }^{j}$ Siratori Clinic, Tokyo, ${ }^{k}$ Department of Nephrology and Hypertension, Internal Medicine, St. Marianna University School of Medicine, Kawasaki, 'Department of Nephrology, Hypertension, Diabetology, Endocrinology and Metabolism, Fukushima Medical University School of Medicine, Fukushima, ${ }^{m}$ Division of Nephrology, Kanazawa Medical University School of Medicine, Uchinada, n'Department of Clinical Pharmacology and Therapeutics, Graduate School of Pharmaceutical Sciences, Tohoku University, Sendai, ${ }^{\circ}$ Department of Laboratory Medicine, Kanazawa University

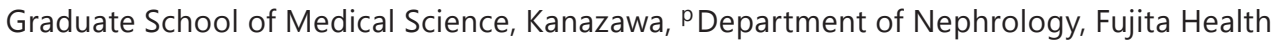
University School of Medicine, Toyoake, ${ }^{9}$ Division of Nephrology and Rheumatology, Department of Internal Medicine, and ' General Medical Research Center, Faculty of Medicine, Fukuoka University, Fukuoka, ${ }^{5}$ Division of Nephrology, Kanayama Clinic, and 'Department of Nephrology, Nagoya University Graduate School of Medicine, Nagoya, "Kodama Hospital, Wakayama, " Hiroshima Kidney Organization, Hiroshima, and w Nakayamadera Imai Clinic, Takarazuka, Japan

\section{Key Words}

LDL apheresis - Drug-resistant nephrotic syndrome - Long-term outcome · Nephrotic syndrome · POLARIS study · Prospective study · Urinary protein

\section{Abstract}

Background/Aims: LDL apheresis (LDL-A) is used for drug-resistant nephrotic syndrome (NS) as an alternative therapy to induce remission by improvement of hyperlipidemia. Several clinical studies have suggested the efficacy of LDL-A for refractory NS, but the level of evidence remains insufficient. A multicenter prospective study, POLARIS (Prospective Observational Survey on the Long-Term Effects of LDL Apheresis on Drug-Resistant Nephrotic Syndrome), 
Muso et al.: A Prospective Observational Survey on the Long-Term Effect of LDL Apheresis on Drug-Resistant Nephrotic Syndrome

was conducted to evaluate its clinical efficacy with high-level evidence. Methods: Patients with NS who showed resistance to primary medication for at least 4 weeks were prospectively recruited to the study and treated with LDL-A. The long-term outcome was evaluated based on the rate of remission of NS 2 years after treatment. Factors affecting the outcome were also examined. Results: A total of 58 refractory NS patients from 40 facilities were recruited and enrolled as subjects of the POLARIS study. Of the 44 subjects followed for 2 years, 21 $(47.7 \%)$ showed remission of NS based on a urinary protein (UP) level $<1.0 \mathrm{~g} /$ day. The UP level immediately after LDL-A and the rates of improvement of UP, serum albumin, serum creatinine, eGFR, and total and LDL cholesterol after the treatment session significantly affected the outcome. Conclusions: Almost half of the cases of drug-resistant NS showed remission 2 years after LDL-A. Improvement of nephrotic parameters at termination of the LDL-A treatment was a predictor of a favorable outcome.

\section{Introduction}

In nephrotic syndrome (NS), plasma proteins are lost in urine, and compensating protein synthesis including lipoprotein evokes a hypercholesterolemic condition. Persistent hypercholesterolemia may promote progressive renal injury [1]; thus, hypercholesterolemia is not only a complication, but also an exacerbating factor. Therefore, rapid normalization of hypercholesterolemia is important for prevention of progression of renal injury in NS.

LDL apheresis (LDL-A) is a blood purification therapy that removes LDL from the circulating blood flow and rapidly reduces the plasma cholesterol level. LDL-A was originally developed for treatment of serious hyperlipidemia, such as familial hypercholesterolemia that is unresponsive to ordinary lipid-lowering drugs [2]. Subsequently, LDL-A began to be used to improve dyslipidemia in NS (initially to prevent renal injury) and was found to improve nephrotic symptoms, including the level of urinary protein (UP) and hyperproteinemia [3], in addition to ameliorating dyslipidemia.

Several studies in patients with NS have shown relatively favorable therapeutic effects even for refractory types of NS such as focal segmental glomerulosclerosis (FSGS), and not only in adults [4-7], but also in pediatric patients [8,9]. However, the evidence levels of these studies have been relatively low, because most were retrospective analyses or had a small number of subjects. To establish more convincing evidence for the efficacy of LDL-A, we performed a prospective cohort study, POLARIS (Prospective Observational Survey on the Long-Term Effects of LDL Apheresis on Drug-Resistant Nephrotic Syndrome), in which patients with NS that were unresponsive to primary medication were prospectively recruited and followed up for 2 years after LDL-A treatment. We have preliminarily analyzed the effect of LDL-A on recovery from NS immediately after treatment and revealed a rapid normalization of proteinuria in approximately half of the subjects [10]. In the current report, we evaluated the 2-year outcome of the same cohort and examined factors that affect clinical efficacy as the final results of the POLARIS study.

\section{Subjects and Methods}

\section{Study Design and Subjects}

The POLARIS study is a prospective, observational, multicenter cohort study based on a central registration system. The study protocol was approved by the Institutional Review 
Muso et al.: A Prospective Observational Survey on the Long-Term Effect of LDL Apheresis on Drug-Resistant Nephrotic Syndrome

Boards at Fukuoka University Hospital, Fukuoka, and the Tazuke Kofukai Medical Research Institute, with which the core investigators (T. Saito and E. Muso) are affiliated. The study is also registered in the University Medical Information Network, Japan (ID: UMIN000000871).

Patients with NS resistant to primary medication for at least 4 weeks after drug treatment had first been administered were prospectively registered as subjects in the POLARIS study from January 1, 2007, to December 31, 2008. Each subject was informed about the outline of the study as well as the risks and benefits of LDL-A before enrollment and then provided written informed consent before registration in the study. The overall study was conducted in accordance with the Declaration of Helsinki.

This study was planned to be conducted as an observational study intended to reveal the actual efficacy of LDL-A in combination with standard therapy in ordinary clinical practice. Therefore, the study protocol does not specify each patient's treatment regimen, leaving this to the attending physician's discretion. The laboratory data were measured at each treatment site.

\section{Evaluation of Outcome}

The 2-year outcome after LDL-A was evaluated by the attending doctor of each subject using a 4-category scale that has been shown to be effective in Japan [11]: complete remission = UP undetectable; incomplete remission I = UP $<1.0 \mathrm{~g} /$ day; incomplete remission II = UP $\geq 1.0$ to $<3.5 \mathrm{~g} /$ day, and no effect $=U P \geq 3.5 \mathrm{~g} /$ day. In this study, complete remission or incomplete remission I was defined as a favorable outcome, and the other categories as a poor outcome. The rate of subjects with a favorable outcome was evaluated 2 years after the LDL-A treatment.

\section{Results}

\section{Subject Background and Clinical Characteristics}

As shown in figure 1, 58 patients with NS were initially registered, but 5 were excluded due to unavailability of background data $(n=3)$ and violation of inclusion criteria $(n=2)$. During the 2 years of follow-up, 3 died of diseases unrelated to NS and 6 were lost to followup, resulting in 44 subjects analyzed in the current study. Although the type of LDL-A modality was not specified in the protocol, the dextran sulfate adsorption system (Liposorber ${ }^{\circledR}$; Kaneka Corporation, Osaka, Japan) was used in all cases. The average number of LDL-A sessions in each LDL-A treatment series was $9.6 \pm 2.7$. The average volume of treated plasma in each session was $3.5 \pm 0.8$ liters. Other characteristics of the patients and the LDL-A treatment are also described in table 1 . As for primary diseases of the subjects, FSGS was found in the majority of cases, presenting in 28 subjects (63.6\%), which presumably reflects the actual situation of clinical practice. The other primary diseases were: membranous nephropathy ( $n=4)$; minimal change NS (MCNS), renal amyloidosis, and undescribed cases ( $n=2$ each), and lupus nephritis, membranoproliferative glomerulonephritis, Henoch-Schönlein purpura nephritis, crescentic glomerulonephritis, diabetic nephropathy, and hepatitis B virusassociated nephropathy ( $\mathrm{n}=1$ each).

\section{Outcomes at the 2-Year Follow-Up}

During the follow-up period, 3 subjects died of diseases unrelated to NS (cerebral infarction, lung cancer, and methicillin-resistant Staphylococcus aureus pneumonia) and 6 were lost to follow-up, leaving 44 subjects eligible for analysis at the 2-year follow-up (fig. 1). A favorable outcome was found in $47.7 \%(21 / 44 ; 11$ subjects in complete remission and 10 in incomplete remission I), whereas 23 subjects had an unfavorable outcome (11 in incomplete remission II and 12 with no effect) (fig. 2). 
nephron

Fig. 1. Flowchart of enrollment and follow-up of patients registered in the study. A total of 58 patients were enrolled in the POLARIS study. However, 5 of them were excluded because LDL-A treatment was started within 4 weeks of drug treatment in 2 patients and the primary data including clinical parameters before and immediately after treatment were not collected in 3 patients. Thus, 53 patients were followed up for 2 years as the subjects of the study. During the follow-up period, 3 died of diseases unrelated to NS (cerebral infarction, lung cancer, and methicillinresistant $S$. aureus pneumonia) and 6 were lost to follow-up, leaving 44 subjects for evaluation of 2-year outcomes.

Table 1. Subject background and treatment characteristics

\begin{tabular}{l|l}
\hline Nephron Extra 2015;5:58-66 \\
\hline DOI: 10.1159/000437338 & $\begin{array}{l}\text { @ } 2015 \text { The Author(s). Published by S. Karger AG, Basel } \\
\text { www.karger.com/nne }\end{array}$ \\
\hline
\end{tabular}

Muso et al.: A Prospective Observational Survey on the Long-Term Effect of LDL Apheresis on Drug-Resistant Nephrotic Syndrome

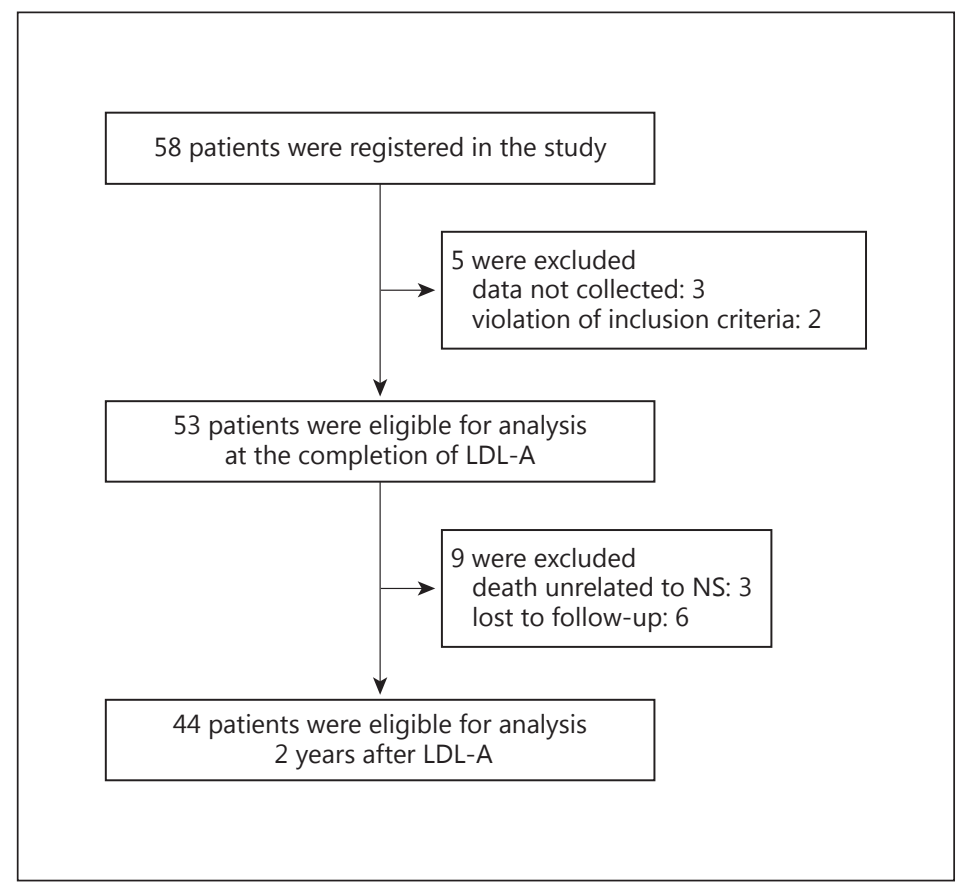

\begin{tabular}{lc}
\hline Characteristics & Value \\
\hline Subjects & 53 \\
Age (range), years & $55.8 \pm 18.1(18-87)$ \\
Male/female & $33 / 20$ \\
First time/recurrent & $31 / 21^{\mathrm{a}}$ \\
Renal biopsy (+/-) & $44 / 8^{\mathrm{a}}$ \\
Concomitant administration of & \\
$\quad$ cyclosporine A (+/-) & $24 / 27^{\mathrm{b}}$ \\
Concomitant administration of & \\
$\quad$ steroid pulse therapy (+/-) & $4 / 47^{\mathrm{b}}$ \\
Number of LDL-A sessions & $9.6 \pm 2.7$ \\
Treated amount of plasma, l & $3.5 \pm 0.8$ \\
\hline
\end{tabular}

Values are presented as means \pm SD or $n$.

${ }^{\mathrm{a}}$ Data not collected for 1 subject. ${ }^{\mathrm{b}}$ Data not collected for 2 subjects.

\section{Factors Affecting Outcome}

The subjects' background factors, including age, sex, BMI, primary or recurrent cases, concomitant calcineurin inhibitor administration, concomitant steroid pulse therapy, and the number of LDL-A sessions, were not significantly correlated with outcome. Most serum and urine parameters before and after treatment also showed no significant correlation. However, the UP level immediately (within up to 4 weeks) after LDL-A was strongly associated with 2 -year outcome $(\mathrm{p}<0.0001)$ (table 2). Of the 20 subjects relieved from nephrotic-range proteinuria with a UP level of $<3.5 \mathrm{~g} /$ day immediately after LDL-A, $16(80.0 \%)$ had favorable outcomes, whereas only 4 of the 22 subjects $(18.2 \%)$ without posttreatment relief from NS achieved favorable outcomes ( $\mathrm{p}<0.001, \chi^{2}$ test).

Correlations between 2-year outcome and change of serum and urine parameters before and immediately after LDL-A treatment were also examined. This analysis revealed that the 
Muso et al:: A Prospective Observational Survey on the Long-Term Effect of LDL Apheresis on Drug-Resistant Nephrotic Syndrome

Table 2. Mean values of serum and urine parameters immediately after LDL-A treatment in subjects with favorable and poor outcomes

\begin{tabular}{lccccc}
\hline Clinical parameter & $\begin{array}{l}\text { Favorable } \\
\text { outcome }\end{array}$ & $\mathrm{n}$ & $\begin{array}{l}\text { Poor } \\
\text { outcome }\end{array}$ & $\mathrm{n}$ value \\
\hline Serum total protein, g/dl & $4.9 \pm 0.7$ & 21 & $4.6 \pm 0.8$ & 23 & 0.230 \\
Serum albumin, g/dl & $2.9 \pm 0.8$ & 21 & $2.5 \pm 0.7$ & 23 & 0.061 \\
Serum creatinine, mg/dl & $1.2 \pm 0.7$ & 21 & $2.0 \pm 2.0$ & 23 & 0.092 \\
eGFR, ml/min/m & $61.0 \pm 27.2$ & 21 & $46.6 \pm 28.2$ & 22 & 0.095 \\
UP, g/day & $1.7 \pm 1.8$ & 20 & $6.2 \pm 3.5$ & 22 & $<0.0001$ \\
Triglycerides, mg/dl & $240.2 \pm 156.3$ & 19 & $241.6 \pm 214.0$ & 19 & 0.981 \\
Total cholesterol, mg/dl & $194.3 \pm 65.6$ & 20 & $253.1 \pm 157.0$ & 22 & 0.128 \\
LDL cholesterol, mg/dl & $83.1 \pm 60.4$ & 18 & $115.1 \pm 58.9$ & 21 & 0.103 \\
HDL cholesterol, mg/dl & $66.5 \pm 18.3$ & 18 & $78.1 \pm 28.7$ & 17 & 0.162 \\
Fibrinogen, mg/dl & $271.1 \pm 77.2$ & 15 & $354.8 \pm 130.7$ & 10 & 0.055 \\
Thrombin-antithrombin III complex, ng/ml & $14.7 \pm 38.6$ & 14 & $3.3 \pm 3.1$ & 6 & 0.488 \\
\hline
\end{tabular}

Fig. 2. Remission rate at 2 years after LDL-A treatment. The outcomes 2 years after LDL-A treatment for 44 subjects were evaluated with a 4-category scale as described in the Subjects and Methods section. Complete remission or incomplete remission I was defined as a favorable outcome, and incomplete remission II or no effect as a poor outcome. The rate of favorable outcomes was $47.7 \%$ (21/44).

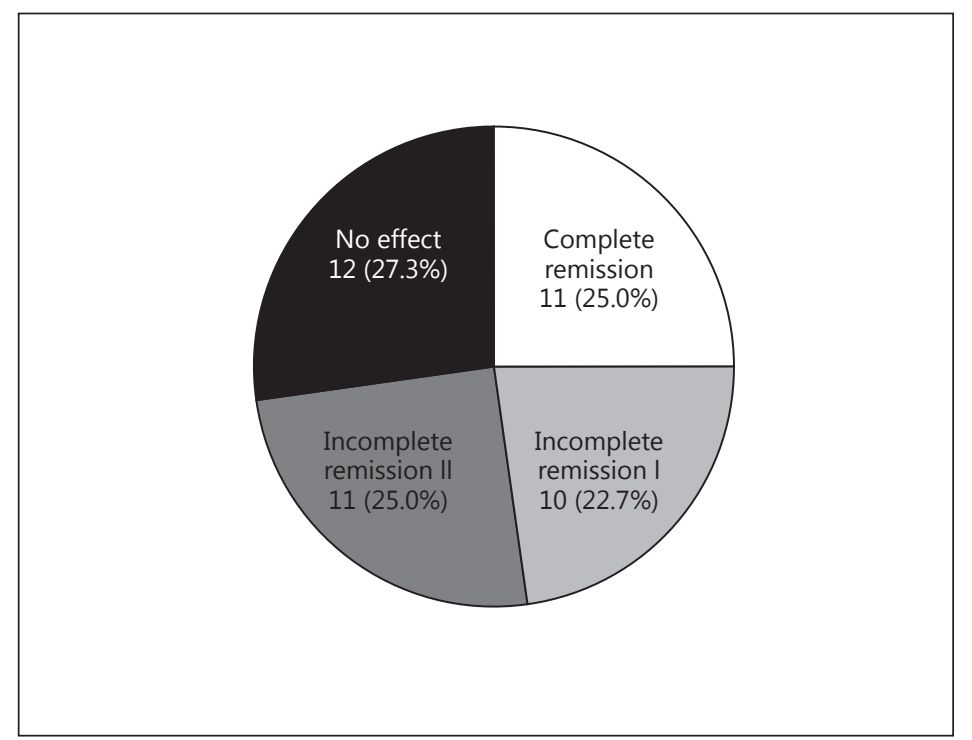

rates of improvement of serum albumin, eGFR, UP, and total and LDL cholesterol were significantly higher in subjects with a favorable outcome (table 3). Regarding primary diseases, the 2 subjects with MCNS had a favorable outcome, and the 2 subjects with renal amyloidosis had a poor outcome, but other primary diseases showed no correlation with outcome. In addition, the subjects with non-FSGS in this study on the whole showed slightly better outcomes than those with FSGS (table 4).

\section{Discussion}

The POLARIS study was conducted as a prospective evaluation of the response to and outcome of LDL-A treatment in patients with NS who had a poor response to primary medication in an actual clinical setting. We had reported that LDL-A had a short-term clinical 
Muso et al.: A Prospective Observational Survey on the Long-Term Effect of LDL Apheresis on Drug-Resistant Nephrotic Syndrome

Table 3. Percent change of serum and urine parameters before and immediately after LDL-A treatment in subjects with favorable and poor outcomes

\begin{tabular}{lrrrrr}
\hline Clinical parameter & $\begin{array}{l}\text { Favorable } \\
\text { outcome }\end{array}$ & $\mathrm{n}$ & \multicolumn{1}{l}{$\begin{array}{l}\text { Poor } \\
\text { outcome }\end{array}$} & $\mathrm{n}$ & $\mathrm{p}$ value \\
& $9.9 \pm 21.0$ & 19 & $8.7 \pm 12.9$ & 22 & 0.816 \\
Serum total protein & $39.5 \pm 34.4$ & 21 & $16.2 \pm 20.5$ & 23 & 0.009 \\
Serum albumin & $-17.8 \pm 26.1$ & 21 & $3.8 \pm 39.1$ & 23 & 0.038 \\
Serum creatinine & $46.4 \pm 78.9$ & 21 & $8.4 \pm 34.7$ & 22 & 0.046 \\
eGFR & $-62.2 \pm 57.1$ & 20 & $-11.7 \pm 44.4$ & 22 & 0.003 \\
UP & $-0.9 \pm 50.2$ & 19 & $1.5 \pm 65.5$ & 19 & 0.901 \\
Triglycerides & $-42.0 \pm 19.7$ & 20 & $-15.9 \pm 46.5$ & 22 & 0.026 \\
Total cholesterol & $-59.6 \pm 27.4$ & 18 & $-31.4 \pm 41.0$ & 20 & 0.019 \\
LDL cholesterol & $0.44 \pm 25.51$ & 18 & $15.89 \pm 24.89$ & 15 & 0.090 \\
HDL cholesterol & $-18.97 \pm 26.78$ & 15 & $-6.32 \pm 31.02$ & 10 & 0.288 \\
Fibrinogen & $-19.04 \pm 64.79$ & 13 & $-42.95 \pm 39.08$ & 5 & 0.456 \\
Thrombin-antithrombin III complex & & & & & \\
\hline
\end{tabular}

Table 4. Frequency of primary diseases in subjects with favorable and poor 2-year outcomes

\begin{tabular}{|c|c|c|c|c|}
\hline \multirow{2}{*}{$\frac{\text { Disease }}{\text { FSGS }}$} & \multicolumn{2}{|c|}{ Favorable outcome } & \multicolumn{2}{|c|}{ Poor outcome } \\
\hline & $12^{\mathrm{a}}$ & $42.9 \%$ & $16^{\mathrm{b}}$ & $57.1 \%$ \\
\hline Non-FSGS & 7 & $50.0 \%$ & 7 & $50.0 \%$ \\
\hline MN & 1 & & 3 & \\
\hline MCNS & 2 & & 0 & \\
\hline RA & 0 & & 2 & \\
\hline Others & $4^{c}$ & & $2^{\mathrm{d}}$ & \\
\hline Not described & 2 & & 0 & \\
\hline Total & 21 & $47.7 \%$ & 23 & $52.3 \%$ \\
\hline \multicolumn{5}{|c|}{$\begin{array}{l}\text { MN = Membranous nephropathy; RA = renal amyloidosis. } \\
{ }^{\mathrm{a}} \text { Including } 1 \text { subject complicated with another renal disease (MN). } \\
\text { Including } 2 \text { subjects complicated with other renal diseases (MN: } 1 \text {; } \\
\text { diabetic nephropathy: } 1 \text { ). }{ }^{\mathrm{c}} \text { Lupus nephritis: } 1 \text {; crescentic glomerulo- } \\
\text { nephritis: 1; diabetic nephropathy: } 1 \text {; hepatitis B virus-associated ne- } \\
\text { phropathy: } 1 \text {. }^{\mathrm{d}} \text { Membranoproliferative glomerulonephritis: } 1 \text {; Henoch- } \\
\text { Schönlein purpura nephritis: } 1 \text {. }\end{array}$} \\
\hline
\end{tabular}

efficacy, with relief from NS as early as 4 weeks after treatment in approximately half of the treated cases [10]. In this study, we examined the long-term outcome and found that nearly half of the subjects $(21 / 44 ; 47.7 \%)$ had relief from NS 2 years after treatment. These results suggest that the therapeutic efficacy of LDL-A immediately after treatment was largely maintained for 2 years.

We had previously conducted a retrospective survey to examine the long-term outcome of patients with NS treated with LDL-A and found a favorable outcome rate of $62.1 \%(18 / 29)$ [7], which is considerably higher than that in the current prospective study. This discrepancy may be due to selection bias, because the subjects in the retrospective study were selected from cases reported in journals or at conferences. Cases selected for presentation tend to be biased toward favorable results. The current study is important in that it determines the outcome prospectively after LDL-A in patients with NS who were unresponsive to ordinary medication. 
Muso et al.: A Prospective Observational Survey on the Long-Term Effect of LDL Apheresis on Drug-Resistant Nephrotic Syndrome

Several reports have evaluated the efficacy of LDL-A for non-FSGS diseases, such as MCNS [12], membranous nephropathy [13,14], lupus nephritis [15-17], and diabetic nephropathy [18-21], but most are case reports/series with a limited number of subjects. Although the number of subjects with each disease apart from FSGS is small, we examined a total of 16 subjects with non-FSGS registered in the POLARIS cohort and found that LDL-A treatment reduced the UP level in those patients at a comparable rate to that in FSGS cases immediately after treatment [10]. In the current study, we were able to examine a total of 14 non-FSGS subjects and showed that the rate of favorable outcomes was $50.0 \%$, which is comparable to - or even better than - the rate in FSGS subjects, reflecting the immediate proteinuria-reducing effect (table 4). This suggests that therapeutic mechanisms of LDL-A may be involved in some factors common to NS.

On the basis of reviewing previous reports, several mechanisms could be proposed to explain the therapeutic effects of LDL-A: improvements in glomerular injuries, which are likely to be caused by activated macrophages through amelioration of dyslipidemia [22, 23]; directadsorption and removal of circulating noxious factors (e.g., coagulation factors including fibrinogen and von Willebrand factor [24, 25]), and recovery of the drug potency of lipophilic drugs, such as cyclosporine, the availability of which is impaired in the hyperlipidemic condition $[26,27]$.

In this study, factors that could affect the outcome of the subjects were examined. No baseline clinical parameters and conditions (i.e., serum and urine parameters before treatment and subject background) examined in this study showed a significant correlation with 2-year outcome, and most serum and urine parameters after treatment were not correlated with outcome. However, the UP levels after treatment differed significantly between subjects with favorable and those with poor outcomes, with mean values of $1.68 \pm 1.76$ and $6.18 \pm 3.14 \mathrm{~g} /$ day (table 2), respectively; furthermore, $80 \%$ of the subjects who achieved posttreatment relief from NS (UP $<3.5 \mathrm{~g} /$ day) had a favorable outcome. Several studies have also shown that proteinuria in the nephrotic range is associated with a poor prognosis and onset of end-stage renal disease over several years, while recovery from nephrotic proteinuria portends a favorable outcome for several types of NS, including FSGS [28, 29], membranous nephropathy [30, 31], and IgA nephropathy [32,33]. Improvement of parameters representing disease conditions of NS, including serum albumin, eGFR, UP, and total and LDL cholesterol, was also significantly associated with favorable outcome (table 4), which suggests that an early rapid alleviation of pathological conditions of NS by LDL-A contributes to a favorable outcome.

Summing up the results obtained in the POLARIS study, it is conceivable that, regardless of the type of primary disease, LDL-A rapidly ameliorated proteinuria in nearly half of the patients with NS who failed to respond to primary medication and that most of these patients then had a favorable 2-year outcome. Although this study was conducted as a single-arm trial with no controls, the efficacy of LDL-A is considered to be fairly convincing, since it is well known that drug-resistant patients with NS generally have a poor prognosis.

We recognize that there are several limitations to this study. Most importantly, the study was performed as a single-arm observational study with no control group, and the sample size of 44 was relatively small for a definitive evaluation of treatment efficacy and outcome. However, the study produced quantitative results that reflect the clinical efficacy of LDL-A in actual clinical practice. We consider that LDL-A treatment exerts a rapid amelioration of nephrotic conditions (especially proteinuria) and ensures a favorable outcome in nearly half of drug-resistant patients with NS regardless of the primary disease. Further investigation with a larger number of subjects and preferably controls is needed to confirm the findings of the current study. 
Muso et al:: A Prospective Observational Survey on the Long-Term Effect of LDL Apheresis on Drug-Resistant Nephrotic Syndrome

\section{Acknowledgements}

This study was supported by the Research and Surveillance Group for Progressive Renal Impairment of the Ministry of Health, Labor and Welfare of Japan. The authors express their appreciation to all the investigators who reported clinical data in this study, including Atsushi Oyama (Nishi-Kobe Medical Center), Noriaki Henmi (Fukaya Red Cross Hospital), Noriko Mori (Shizuoka General Hospital), Osamu Nishi (Nishi Clinic), Yasukiyo Mori (Kyoto Prefectural University of Medicine), Megumu Fukunaga (Toyonaka Municipal Hospital), Masahiko Miyamoto (Osaka Red Cross Hospital), Kenji Arizono (Kumamoto Chuo Hospital), Takako Suzuki (Moriyama Rehabilitation Hospital), Kazuhiko Hora (Hokushin General Hospital), Hiroshi Makino (Okayama University), Hideyasu Kiyomoto (Kagawa University Medical School), Yutaka Ando (Osaka Minami Medical Center), Yoshiharu Tsubakihara (Osaka General Medical Center), Kosuke Ota (Okayama Medical Center), Masamichi Fukuda (Iwakuni Medical Center Hospital), Yukiko Abe (Shin-Oura Hospital), Mitsuhiro Yoneda (Fuke Chiba Hospital), Hiroshi Ohtani (Akita Kumiai General Hospital), Tokuichiro Sugimoto (Mitsui Memorial Hospital), Shizunori Ichida (Japanese Red Cross Nagoya Daiichi Hospital), Kentaro Wada (Nippon Kokan Fukuyama Hospital), Rhosuke Yoshihara (Konan Kakogawa Hospital), Shoichi Fujimoto (University of Miyazaki), Morihiro Kondo (Rakuwa-kai Otowa Hospital), Takeshi Nakanishi (Hyogo College of Medicine), Kazuhiko Tsuruya (Kyusyu University), Fumiki Tanigawa (Shimonoseki Kosei Hospital), Masami Hashimoto (Onomichi General Hospital), Akiko Nakamura (Saga Prefectural Hospital Koseikan), Soshi Yorifuji (Osaka Saiseikai Nakatsu Hospital), and Tamaki Sasaki (Kawasaki Medical School).

\section{Disclosure Statement}

This study was conducted under a grant from the Kidney Foundation, Japan, which was financially supported by Kaneka Corporation (Osaka, Japan).

\section{References}

$>1$ Moorhead JF, Chan MK, El-Nahas M, Varghese Z: Lipid nephrotoxicity in chronic progressive glomerular and tubulo-interstitial disease. Lancet 1982;2:1309-1311.

-2 Yokoyama S, Hayashi R, Satani M, Yamamoto A: Selective removal of low density lipoprotein by plasmapheresis in familial hypercholesterolemia. Arteriosclerosis 1985;5:613-622.

3 Tojo K, Sakai S, Miyahara T: Possible therapeutic application of low density lipoprotein apheresis (LDL-A) in conjunction with double filtration plasmapheresis (DFPP) in drug-resistant nephrotic syndrome due to focal glomerular sclerosis (FGS). Nihon Jinzo Gakkai Shi 1988;30:1153-1160.

4 Muso E, Yashiro M, Matsushima M, Yoshida H, Sawanishi K, Sasayama S: Does LDL-apheresis in steroidresistant nephrotic syndrome affect prognosis? Nephrol Dial Transplant 1994;9:257-264.

5 Muso E, Mune M, Fujii Y, Imai E, Ueda N, Hatta K, Imada A, Takemura T, Miki S, Kuwahara T, Takamitsu Y, Tsubakihara Y; Kansai FGS LDL Apheresis Treatment (K-FLAT) Study Group: Significantly rapid relief from steroid-resistant nephrotic syndrome by LDL apheresis compared with steroid monotherapy. Nephron 2001; 89:408-415.

6 Yokoyama K, Sakai S, Sigematsu T, Takemoto F, Hara S, Yamada A, Kawaguchi Y, Hosoya T: LDL adsorption improves the response of focal glomerulosclerosis to corticosteroid therapy. Clin Nephrol 1998;50:1-7.

7 Muso E, Mune M, Yorioka N, Nishizawa Y, Hirano T, Hattori M, Sugiyama S, Watanabe T, Kimura K, Yokoyama H, Sato H, Saito T: Beneficial effect of low-density lipoprotein apheresis (LDL-A) on refractory nephrotic syndrome (NS) due to focal glomerulosclerosis (FGS). Clin Nephrol 2007;67:341-344.

-8 Hattori M, Chikamoto H, Akioka Y, Nakakura H, Ogino D, Matsunaga A, Fukazawa A, Miyakawa S, Khono M, Kawaguchi H, Ito K: A combined low-density lipoprotein apheresis and prednisone therapy for steroidresistant primary focal segmental glomerulosclerosis in children. Am J Kidney Dis 2003;42:1121-1130.

-9 Kawasaki Y, Suzuki S, Matsumoto A, Takano K, Suyama K, Hashimoto K, Suzuki J, Suzuki H, Hosoya M: Longterm efficacy of low-density lipoprotein apheresis for focal and segmental glomerulosclerosis. Pediatr Nephrol 2007;22:889-892.

10 Muso E, Mune M, Hirano T, Hattori M, Kimura K, Watanabe T, Yokoyama H, Sato H, Uchida S, Wada T, Shoji T, Yuzawa Y, Takemura T, Sugiyama S, Nishizawa Y, Ogahara S, Yorioka N, Sakai S, Ogura Y, Yukawa S, Iino Y, Imai E, Matsuo S, Saito T: Immediate therapeutic efficacy of low-density lipoprotein apheresis for drug-resistant nephrotic syndrome: evidence from the short-term results from the POLARIS study. Clin Exp Nephrol 2015; 19:379-386.

11 Kida H, Asamoto T, Yokoyama H, Tomosugi N, Hattori: Long-term prognosis of membranous nephropathy. Clin Nephrol 1986;25:64-69. 
12 Kobayashi T, Ando Y, Umino T, Miyata Y, Muto S, Hironaka M, Asano Y, Kusano E: Complete remission of minimal-change nephrotic syndrome induced by apheresis monotherapy. Clin Nephrol 2006;65:423-426.

-13 Ideura T, Hora K, Kaneko Y, Yamazaki T, Tokunaga S, Shigematsu H, Kiyosawa K: Effect of low-density lipoprotein-apheresis on nephrotic syndrome due to membranous nephropathy in renal allograft: a case report. Transplant Proc 2000;32:223-226.

14 Sato Y, Tsunoda S, Nozue T, Pan Q, Wakasugi H, Yoshimura A: Low-density lipoprotein apheresis therapy for steroid- and cyclosporine-resistant idiopathic membranous nephropathy. Intern Med 2012;51:2597-2602.

15 Daimon S, Saga T, Nakayama M, Nomura Y, Chikaki H, Dan K, Koni I: Dextran sulphate cellulose columns for the treatment of nephrotic syndrome due to inactive lupus nephritis. Nephrol Dial Transplant 2000;15:235238.

16 Kamijo Y, Kaneko Y, Ichikawa T, Kobayashi N, Koyama T, Kakegawa T, Kamijo H, Kono K, Minami S, Tanaka N, Arakura H, Hirata M, Higuchi M, Kiyosawa K, Hora K: A case of nephrotic syndrome due to lupus nephritis which was controlled with low-density lipoprotein apheresis. Ther Apher 2002;6:459-462.

17 Shiraishi N, Kitamura K, Hayata M, Ogata T, Tajiri-Okamura K, Nakayama Y, Kohda Y, Tomita K, Mukoyama M: Low-density lipoprotein apheresis for proteinuria in lupus nephritis with intraglomerular foam cells containing cholesterol crystals. Am J Kidney Dis 2015;65:490-493.

18 Kobayashi S: LDL-apheresis for diabetic nephropathy: a possible new tool. Nephron 1998;79:505-506.

19 Nakao T, Yoshino M, Matsumoto H, Okada T, Han M, Hidaka H, Shino T, Yamada C, Nagaoka Y, Miyahara T: Low-density lipoprotein apheresis retards the progression of hyperlipidemic overt diabetic nephropathy. Kidney Int Suppl 1999;71:S206-S209.

20 Nakamura T, Kawagoe Y, Ogawa H, Ueda Y, Hara M, Shimada N, Ebihara I, Koide H: Effect of low-density lipoprotein apheresis on urinary protein and podocyte excretion in patients with nephrotic syndrome due to diabetic nephropathy. Am J Kidney Dis 2005;45:48-53.

21 Sato E, Amaha M, Nomura M, Matsumura D, Ueda Y, Nakamura T: LDL-apheresis contributes to survival extension and renal function maintenance of severe diabetic nephropathy patients: a retrospective analysis. Diabetes Res Clin Pract 2014;106:241-246.

22 Schlondorff D: Cellular mechanisms of lipid injury in the glomerulus. Am J Kidney Dis 1993;22:72-82.

23 Diamond JR, Pesek I, McCarter MD, Karnovsky MJ: Altered functional characteristics of rat macrophages during nephrosis. Synergistic effects of hypercholesterolemia. Am J Pathol 1989;135:711-718.

24 Knisel W, Di Nicuolo A, Pfohl M, Müller H, Risler T, Eggstein M, Seifried E: Different effects of two methods of low-density lipoprotein apheresis on the coagulation and fibrinolytic systems. J Intern Med 1993;234:479487.

25 Tasaki H, Tsuda Y, Yamashita K, Toyokawa T, Yashiro A, Osajima A, Nakashima Y, Kuroiwa A: Removal of plasma fibrinogen by LDL apheresis. Jpn J Apheresis 1997;16:263.

26 Ingulli E, Tejani A: Severe hypercholesterolemia inhibits cyclosporin A efficacy in a dose-dependent manner in children with nephrotic syndrome. J Am Soc Nephrol 1992;3:254-259.

-27 Leon C, Jia J, Qiu G, Hill JS, Wasan KM: Modification in low density lipoprotein receptor expression affects cyclosporin A cellular uptake and cytotoxicity. J Pharm Sci 2008;97:2350-2361.

-28 Korbet SM: Clinical picture and outcome of primary focal segmental glomerulosclerosis. Nephrol Dial Transplant 1999;14:68-73.

29 Velosa JA, Holley KE, Torres VE, Offord KP: Significance of proteinuria on the outcome of renal function in patients with focal segmental glomerulosclerosis. Mayo Clin Proc 1983;58:568-577.

-30 Shiiki H, Saito T, Nishitani Y, Mitarai T, Yorioka N, Yoshimura A, Yokoyama H, Nishi S, Tomino Y, Kurokawa K, Sakai H; Research Group on Progressive Renal Diseases in Japan: Prognosis and risk factors for idiopathic membranous nephropathy with nephrotic syndrome in Japan. Kidney Int 2004;65:1400-1407.

-31 Cattran DC, Pei Y, Greenwood CM, Ponticelli C, Passerini P, Honkanen E: Validation of a predictive model of idiopathic membranous nephropathy: its clinical and research implications. Kidney Int 1997;51:901-907.

32 Coppo R, D’Amico G: Factors predicting progression of IgA nephropathies. J Nephrol 2005;18:503-512.

-33 Alamartine E, Sabatier JC, Guerin C, Berliet JM, Berthoux F: Prognostic factors in mesangial IgA glomerulonephritis: an extensive study with univariate and multivariate analyses. Am J Kidney Dis 1991;18:12-19. 